\title{
4D Printing - Revolution or Fad?
}

\author{
Author: Eujin Pei \\ Correspondence email: eujinpei1@gmail.com \\ To cite this: Pei, E. (2014) "4D printing - Revolution or Fad?", Assembly Automation, 34(2) \\ DOI: http://dx.doi.org/10.1108/AA-02-2014-014
}

\begin{abstract}
About the author: Eujin is the Programme Director for the BSc Product Design and BSc Product Design Engineering programmes at Brunel University London. He was a Research Fellow at leading institutions including Loughborough University, Brunel University and the University of Southampton; and was a Visiting Scientist to Vaal University of Technology and Central University of Technology, Free State His professional design consultancy work includes those for BAE Systems Applied Intelligence, Motorola, Inc., LM Ericsson, Sennheiser GmbH \& Co. KG, and Rentokil Initial, of which some products are still manufactured and sold today. He is the Convenor for the International Organisation for Standardisation (ISO) TC261/WG4 group, leading Data Transfer and Design Standards for Additive Manufacturing, as well as holding various positions within ISO committee for Additive Manufacturing standards. Eujin is also a Chartered Technological Product Designer (CTPD) with Institution of Engineering Designers in the UK.
\end{abstract}

\begin{abstract}
This feature article reviews state of the art developments in Additive Manufacture, and in particular 4D Printing. It discusses what it is, what research has been carried out and maps potential applications and its future impact. The paper discusses Tibbits' which has investigated how 3D printed parts could transform and adapt over time, and where self-assemblies could occur by means of local interaction. The paper describes the latest generation of 3D printers that are able to print multi-materials with varying shore hardnesses and in colour, the Hyperform and Kinematic projects that have explored the use of mathematical and computational strategies to support folding techniques for 4D printing. The paper highlights why the US\$855,000 worth of funds from the United States Army Research Office is a hint towards the future of a new generation of technology, about the novel mask-image-projection-based Stereolithography system developed by the University of California, and the Suspended Depositions project where researchers from the Southern California Institute of Architecture have achieved suspension of time and material using this technique. Lastly, the paper discusses how scientists from the University of Colorado and Singapore University of Technology and Design have managed to control both material properties and its laminate architecture for shapes to assume complex configurations; as well as the work by Richard Horne and James Corbett who investigated the potential of low-cost techniques for filament blends.
\end{abstract}

\section{Keywords}

Additive Manufacturing, 4D Printing, Rapid Prototyping, Modular Automation, Design for Assembly 


\section{Structured Abstract}

Purpose of this paper

This feature article reviews state of the art developments in Additive Manufacture, particular 4D Printing. It discusses what it is, what research has been carried out and maps potential applications and its future impact.

Design/methodology/approach

The article first defines additive manufacturing technologies and goes on to describe the state-ofart. Following which the paper examines several case-studies and maps a trend that shows an emergence of $4 D$ printing.

Findings

The case-studies highlight a particular specialization within additive manufacture where the use of adaptive, biomimetic composites can be programmed to re-shape, or have embedded properties or functionality that transform themselves when subjected to external stimuli.

What is original/value of paper

This paper discusses the state-of-art of additive manufacture, discussing strategies that can be used to reduce the print process (such as through kinematics); and the use of smart materials where parts adapt themselves in response to the surrounding environment supporting the notion of selfassemblies. 


\subsection{The Rise of Additive Manufacturing}

Additive manufacturing has been around for nearly three decades ever since the first systems were commercially available in the late 1980s. In this process, parts are built a layer at a time, and are in theory not constrained by the complexity of the CAD geometry. The term "solid freeform fabrication" is still used today to describe this process. Parts produced from additive manufacture have been applied in a variety of ways such as in new product development for visual prototypes and functional testing; as well as in medical and aerospace industries for specialised end-use parts.

In line with the increased demand, the number and variety of additive manufacturing systems is set to rise. According to statistics from the Wohler's report, the global market for additive manufacturing products and services in 2012 grew by $28.6 \%$ to US\$2.204 billion (Wohler, 2013). Sales of professional grade systems (those costing more than US\$5,000) rose by $19.3 \%$ to 777,1 units in 2012. At the same time, low-cost personal systems averaged at $346 \%$ each year from 2008 to 2011 and cooled to $46.3 \%$ in 2012 . The popularity of entry-level 3D printers is becoming more affordable for mainstream use and for education with the aim of democratising production for the masses. This new age of personalisation and customisation promises to transform the way things will be produced, either in the comfort of the home or using a Makerspace hub or Fablab that provides access to small-scale manufacturing facilities. The end result is often a socially manufactured product.

\subsection{Time - The Fourth Dimension}

Despite these benefits, one drawback of additive manufactured parts is that they generally tend to be static and inanimate with the exception of specially designed functional and moving parts such as living hinges, integrated ball and socket joints and encapsulated bearings (Maidin et al., 2012). In response to this, a new trend at the other far end of the technology spectrum is emerging. This is time as the fourth dimension, which is combined with 3D printing to be known as " $4 D$ printing". It is not about how long it takes to print a part; but rather the fact that a 3D printed object after being built continues to evolve over time.

What 4D printing offers is the opportunity for objects that change and adapt in response to the surrounding environment. So how is this even possible? The relatively recent advancements of multi-material additive manufacturing, combined with rapid developments in smart materials and active fibres mean that there are now even more opportunities and applications waiting to be discovered. Professor Anna Balazs from the University of Pittsburgh's Swanson School of Engineering describes $4 \mathrm{D}$ printing as the use of adaptive, biomimetic composites that are able to re-shape, or have embedded properties or functionality that transform themselves based on external stimuli. Her research focuses on the computational design of chemo-mechanically responsive gels and composites (University of Pittsburgh, 2013).

Skylar Tibbits who heads the Massachusetts Institute of Technology's Self-Assembly Technologies Lab, is well known for research on 4D printing. Their previous work has focused on the geometry and mechanical computation to understand how synthetic substances can become functional and serving as low-level smart materials (Tibbits and Cheung, 2012). This programmable and computational material, Logic Matter, relies on mechanical computation through the geometric constraints of the build block. At the TED conference held in Los Angeles, Tibbits demonstrated how 4D printed objects could perform self-assemblies where over time static objects transform and adapt. This process is made possible by 3D printing a specialised multi-layered material made up of a strand of standard plastic with a sandwiched layer made from an absorbent substance that expands up to 150 percent when in contact with water. The Stratasys Connex multi-material technology allows such specific material properties to be programmed into controlled areas of the designed geometry (Stratasys, 2013). At this time of writing, the Connex3 Objet500 printers are able to mix 
three different resins in precise ratios within a single print operation, and with a range of 45 possible colours (Figure 1). The system uses synthetic blends that provide different properties, all of which are based on acrylic-based photopolymer resins. These materials are jetted as a liquid and once a layer of material is deposited, it is cured by UV light to form into a solid.

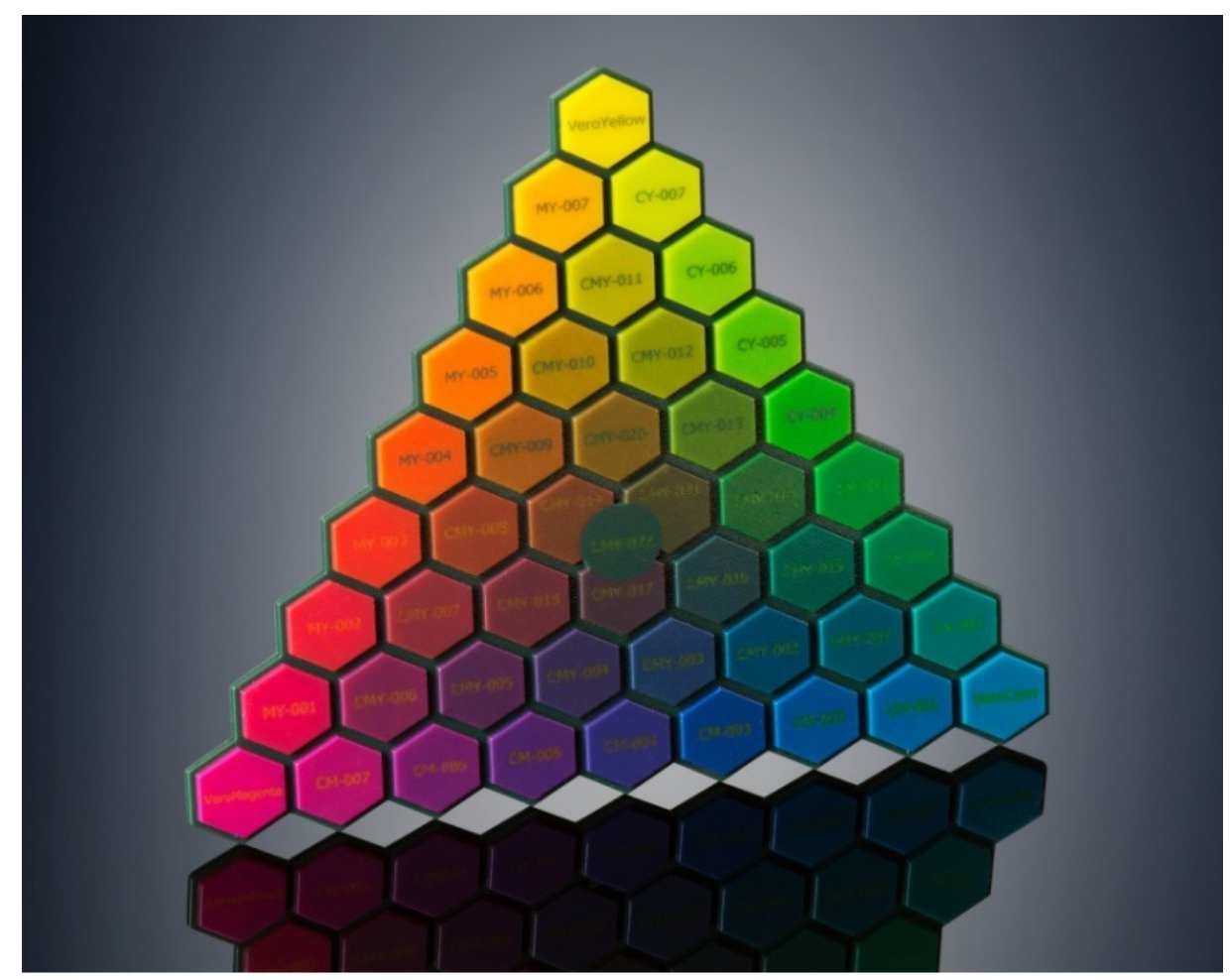

Figure 1: A cyan-magenta-yellow palette from the Objet500 Connex 3 printer (Stratasys, 2014)

Specific materials are chosen so as to harnesses the unique properties that in turn activate the selfassembly process. For example, the expansion of water-absorbing material provides the energy to trigger the bending and twisting action while the remaining material serves as the rigid structure. Tibbits claims that the next stage is to move from printing single strands into sheets, and to eventually build whole structures which he claims as a vision of the future for construction that would tap energy from sources based on heating and expansion, contraction of materials, fluids and capillary action, hydraulics, pneumatics, gravity, magnets, wind resistance, shaking, pre- and posttensional forces or using compressional members such as springs that adapt to changes in the climate, user demands, or loading conditions, etc. Tibbits describes "self-assembly" as a process where disordered parts are built in an ordered structure through local interaction, where the material and geometry must be tightly coupled with the energy source (2012). Project Cyborg from Autodesk Inc., is an example of a simulation software that has been used by researchers to determine how and when the various components will behave during the self-assembly process (Autodesk, 2014).

\subsection{Recent Projects and Developments in 4D Printing}

At an installation at Long Beach, CA. entitled The Self-Assembly Line (Figure 2), pre-fabricated modules are placed in a large rotating chamber (Tibbits and Olson, 2012a). Depending on how quickly the rotating chamber is spun, an interaction takes place between the modules and the end surfaces which contain magnets begin to attract; demonstrating the variables of changing external conditions, the geometry of each component, the attraction mechanism and the number of individual units. It also shows how self-assemblies can occur through time and with the right amount of energy applied to trigger the build process. Such applications can potentially take place in 
extreme environments that encounter weather fluctuations, near-zero gravity situations or in neutral buoyancy, all of which where the indirect energy can spark an interaction. One instance is the use of underwater waves to trigger the self-assembly of structures, or parts dropped from high altitudes that automatically unfold into complete structures.

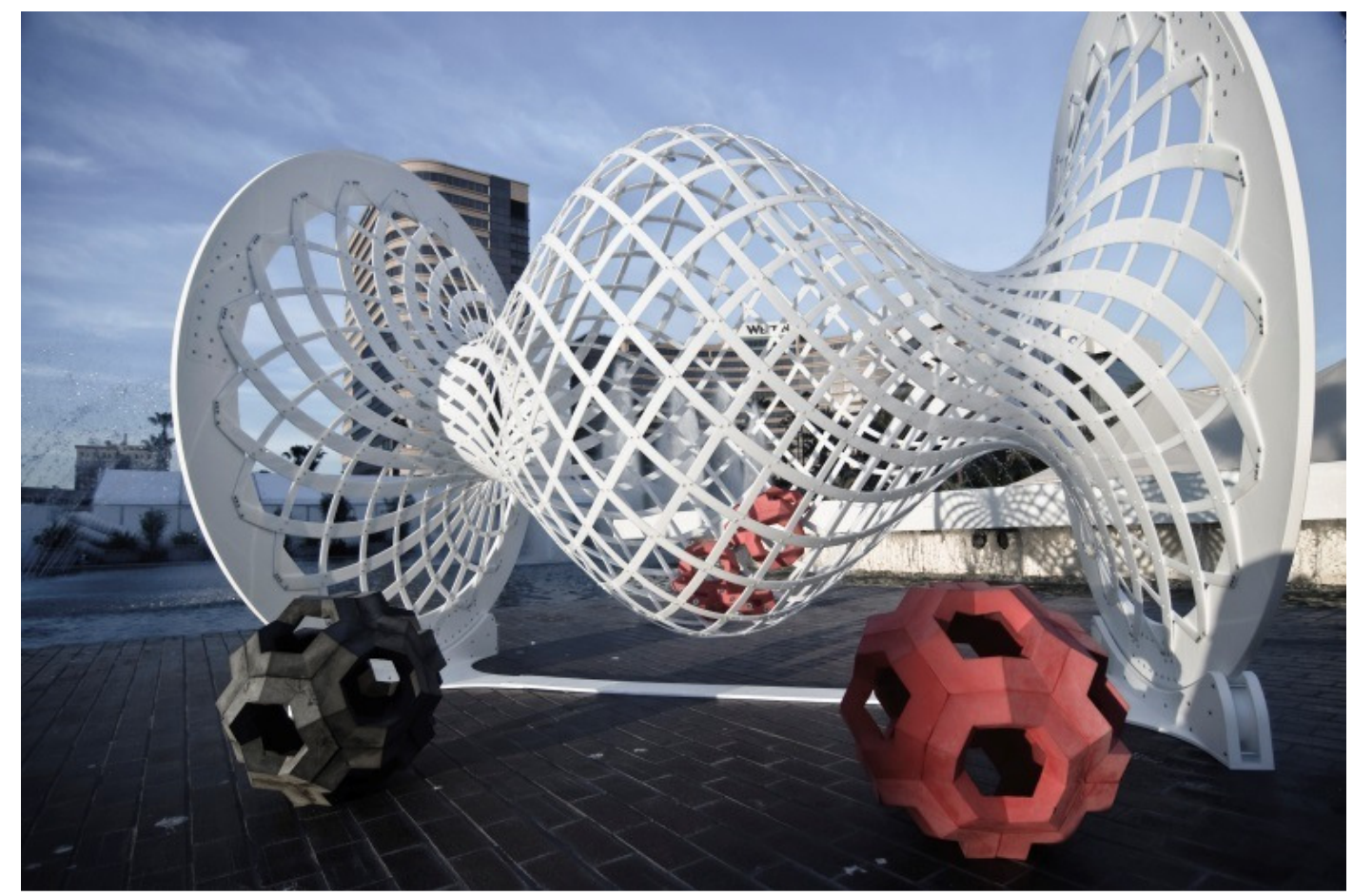

Figure 2: The Self-Assembly Line installation at Long Beach, CA. (Tibbits and Olson, 2012b).

Yet another major limitation of additive manufacturing today is the bed size within the 3D printer that determines the maximum physical dimensions for a part to be built. Although "mammoth" Stereolithography prototyping machines are available, they are still limited by the constraints of the print bed. To counter this, the Hyperform project developed by Marcelo et al. (2013) use mathematical, computational and material folding strategies that enable large scale objects to be compressed into a minimal volume within the print bed, thus maximising the full printing capabilities of the machine. Once printed, the parts unfold and components that contain notches allow it to bend in a certain way to stretch out and extend itself.

Nervous System, a Massachusetts-based design studio led by Jessica Rosenkrantz and Jesse LouisRosenberg (Nervous System, 2013a), has also developed a similar computational method for 3D printing that incorporates articulated joints (Figure 3 ). This allows the built parts to automatically unfold and change their shape once being removed from the build chamber. Named after the science of geometry of motion, they describe the Kinematics project as an example of 4D printing where the finished object is able to self-assemble or transform into a pre-determined form. In this process, they use tessellation software that intelligently splits the CAD model into triangles linked by built-in hinges. Next, the model is computationally folded to compress itself into the smallest possible volume and is optimised to be fabricated within the build space of a 3D printer. They have successfully produced large pieces of dresses and jewellery despite them being much bigger than the size of the print bed. They claim that this holds great promise that will enable the production of other large-scale structures given the constraint of today's small printers. 


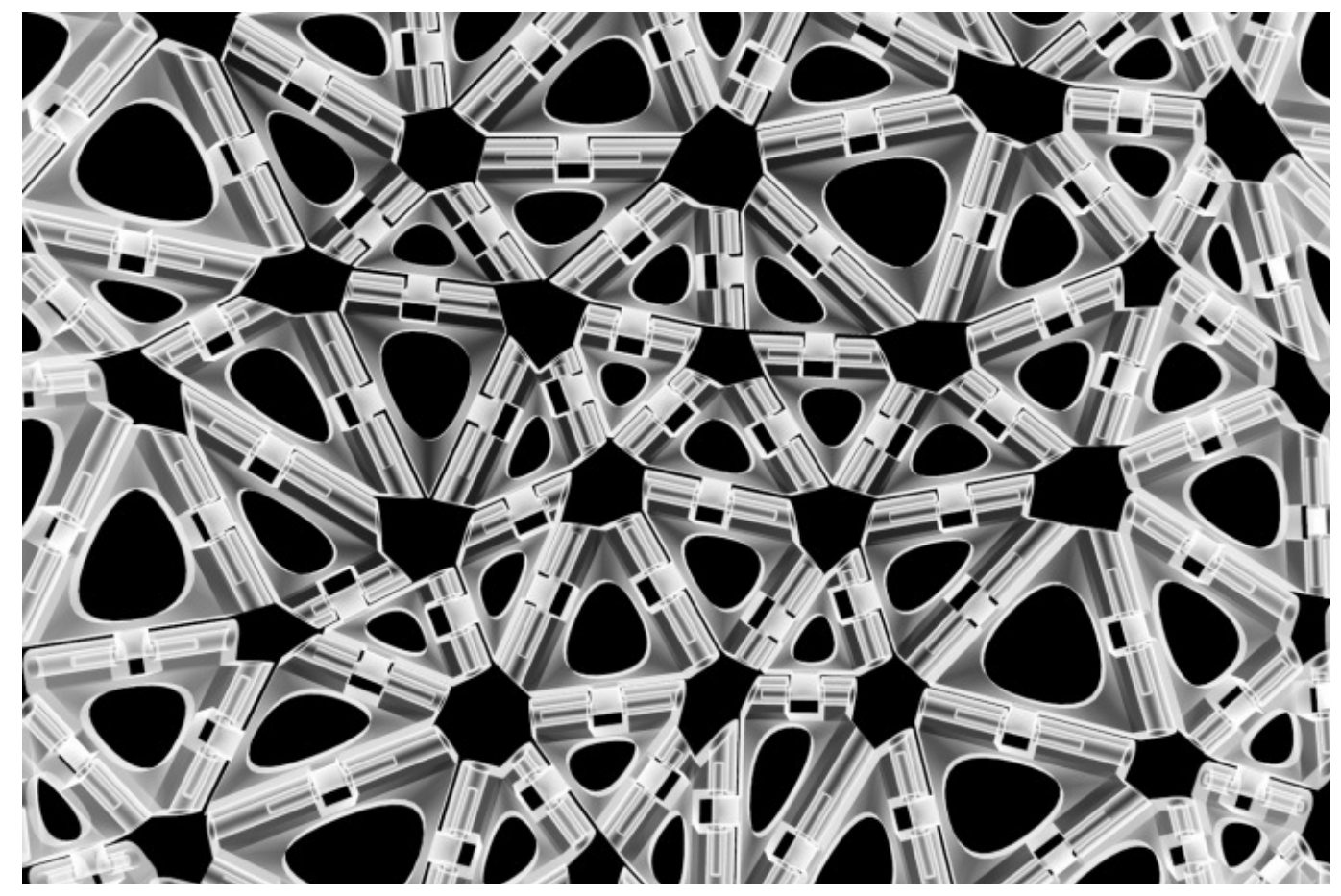

Figure 3: The hinge mechanism from Kinematics showing complex assemblages of jointed parts (Nervous System, 2013b)

The importance of this emerging field of 4D printing has been recognised by the United States Army Research Office who have awarded US\$855,000 worth of funds between three university research teams - Harvard's School of Engineering \& Applied Science, The University of Illinois, and The University of Pittsburgh Swanson School of Engineering, to work on the next generation printing technologies (US Army, 2013). They are looking to investigate the development of adaptive, biomimetic composites that have reprogrammable shapes, properties or function when subject to external stimuli. The area of 3D printing is not new to the organisation where the U.S. Army's Rapid Equipping Force (REF) deployed their second mobile laboratory to the war zone in Afghanistan in 2013. The 20-foot container mobile lab costing US\$2.8 million can be transported to a new location by truck or helicopter. Engineers working inside the lab use 3D printers and CNC systems to machine plastic, steel and aluminium parts when required, thereby accelerating the production process and reducing the need for an inventory especially for small forward operating bases. The Navy is also testing the feasibility of 3D printed ammunition and building unmanned aerial vehicles on board carrier vessels. However, the missing link to the puzzle is the issue of assembly that is generally regarded as being labour intensive, extremely time consuming and requires specialised training. It is hoped that this grant will provide better understanding towards responsive materials, so as to build upon the foundations for the new field of $4 \mathrm{D}$ printing.

Advances in additive manufacturing allow specific materials that possess controlled functional performance to be precisely deposited within a three-dimensional space. Professor Yong Chen and his team from the University of California have developed a novel mask-image-projection-based Stereolithography (MIP-SL) process that claims to reduce the printing time (Pan et al., 2012). They are also exploring the development of a faster multi-material 3D printing process. Using this technique, a CAD model is sliced into a number of horizontal sections, following which a masked image is projected onto the photo-polymer liquid resin that is exposed to UV light for curing. The process is further enhanced by using a two-way projection method that can build a model from both ends or to set multi-material models that require different cure-times. The next step will be to 
examine how to develop an automated system that will position multiple materials according to their specific physical properties.

Scientists from the University of Colorado and Singapore University of Technology and Design have developed an alternative method of building 4D structures. Their work differs from Tibbits' in terms of the physical phenomena at play and greater emphasis on understanding their occurrence. Qi and his team investigated the use of shape-memory polymer composites with glassy fibres to produce 4D parts (Qi et al., 2014). A multi-material composite is printed from a CAD file that specifies the fibre architecture via controllable anisotropic and thermo-mechanical behaviour. By controlling the amount, location and orientation of those fibres, researchers are able to pre-determine how the active composite materials will react when subjected to stimuli such as thermal or mechanical forces that could come from a natural source (e.g. sunlight), from one's body heat or by passing an electric current that will heat up the material. The build process uses shape memory polymer fibres that are sandwiched between an elastomeric matrix. By spatially varying the material properties in a 3D domain and controlling the lamina and the laminate architecture with varying fibre orientations and volume fractions, the reactive layer is able to assume complex three-dimensional configurations such as bending, coiling and twisting, with a dependence of time - the 4D aspect. This concept can be further developed by utilising other functional material properties and using shape and topology optimisation to achieve configuration changes.

The Suspended Depositions project (Figure 4) by Brian Harms and his team from the Southern California Institute of Architecture is a novel process of injecting photo-sensitive resin from a needle print head that is fixed on a robotic arm (Harms et al., 2012a). Strangely, hair gel is used to act as an omnidirectional support material that in turn provides the freedom for the nozzle to navigate and fabricate parts directly on and around other existing components within the gel. The suspension of time (and material) using this technique allows the build process to be paused, for tool change, to modify the digital and physical model, and for multi-material injections within the gel thereby achieving true geometric freedom of manufacture.

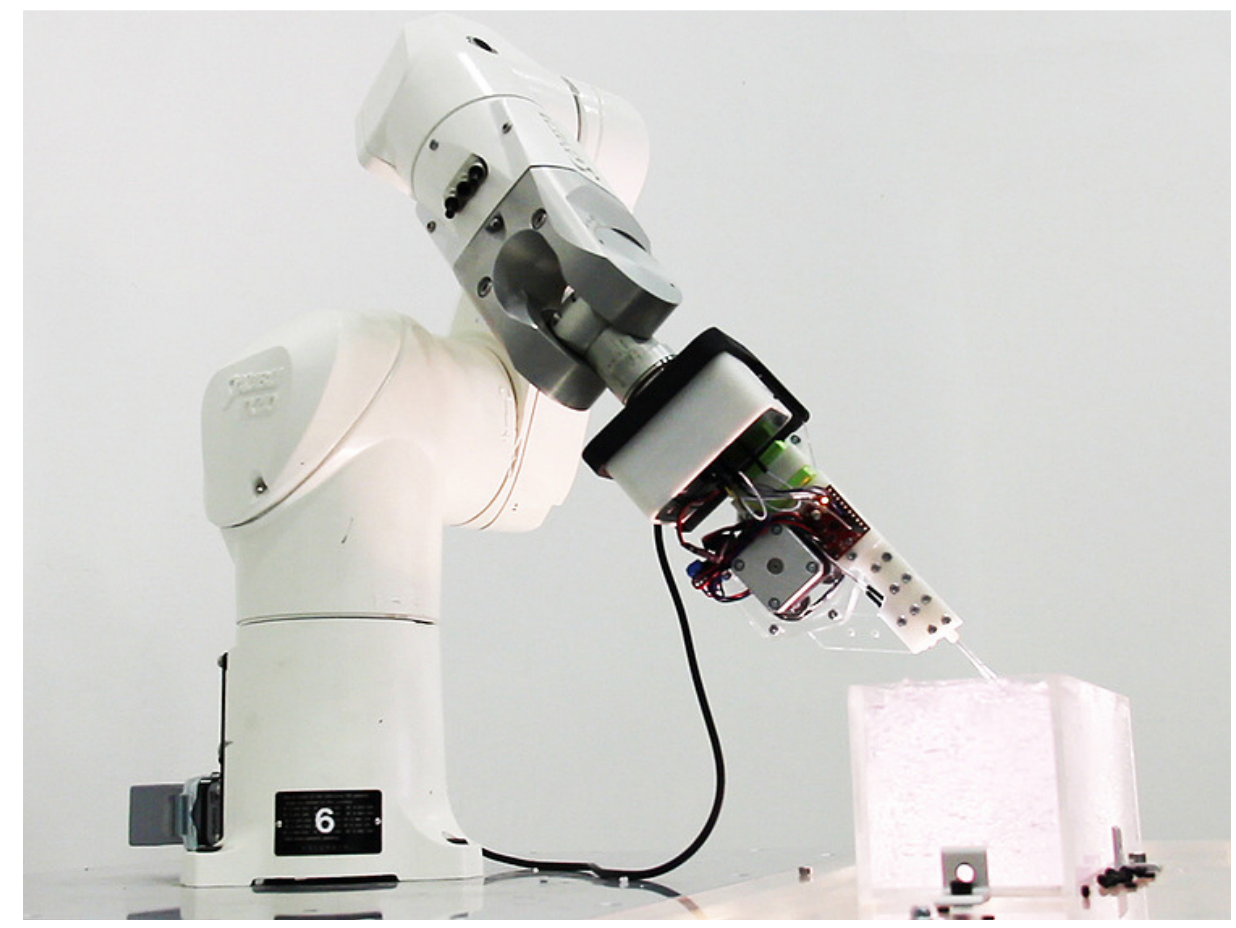

Figure 4: Suspended Deposition looks at suspension of resin in space without added supports (Harms et al., 2012b). 
Much work has also been carried out by DIY enthusiasts such as Richard Horne who uses the moniker of RichRap having developed a colour blending nozzle that in theory could also be used for multi-material printing (Horne, 2012a). Having first experimented with layer selective colour experiments and filament joining, Horne continued to explore the use of single or multiple extruders running at intervals separately or together (figure 5). After several iterations, it was decided that the colour blending nozzle would use a single hot-end with multiple driven feeds that showed promising results. Further work will look at utilising different materials such as PLA for flexible hinges and ABS for structures or even blending different materials together. James Corbett (Corbett, 2012) from the University of Bath, has also explored the feasibility of mixer extruders for Fused Deposition Modelling systems (Figure 5). He has examined the mixing properties of materials and designed an active colour mixing system that uses a hex bar to generate the necessary shear in the viscous plastic for a more homogeneous mixing result. Future work will focus on the development of a software and firmware to gain full independent control of each filament by altering the feeds rates of different colours, and to calibrate a colour space map that will make printing any colour possible.

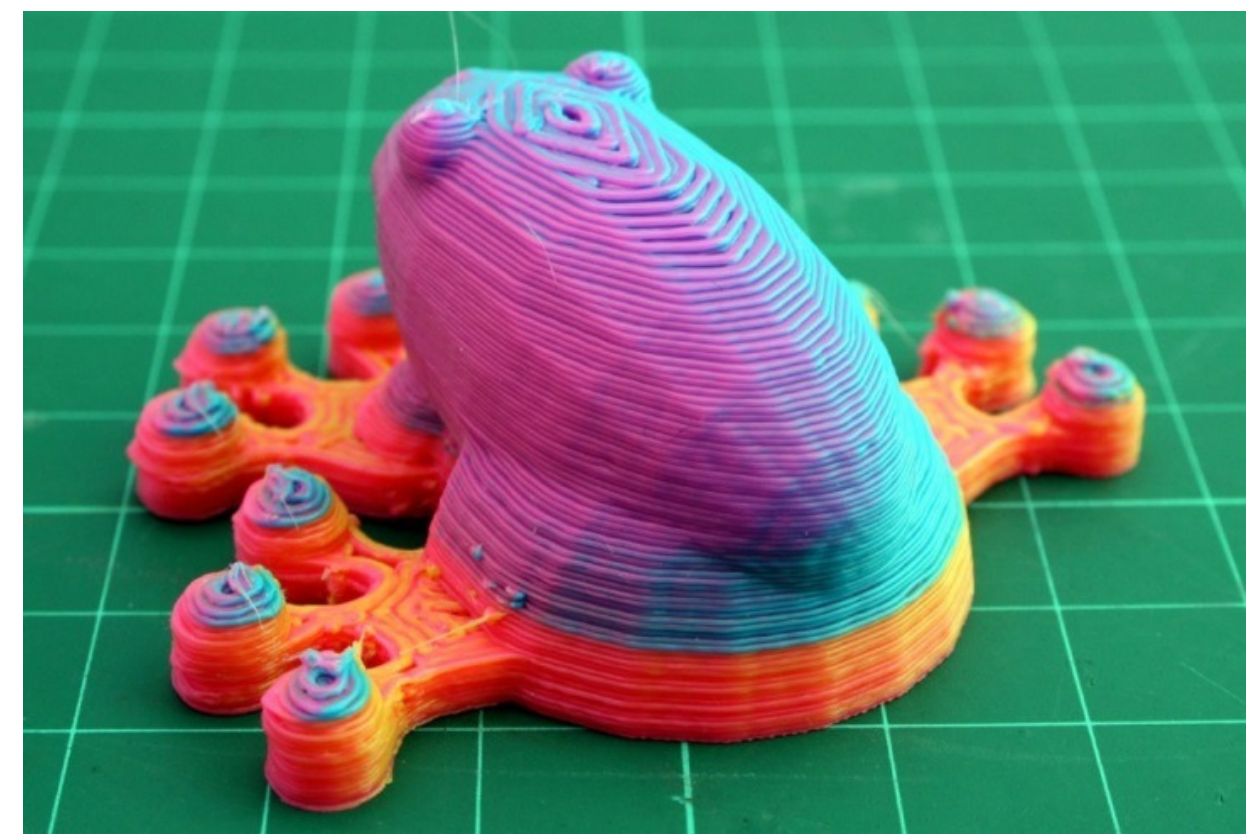

Figure 5: Colour blends from Fused Deposition Modelling (Horne, 2012b).

\subsection{Vision of the Future}

The future of additive manufacturing is set to rise and exciting times are just around the corner. In 2014, key patents that cover laser sintering will begin to expire and we are expected to see an explosion of open-source machines. Radical production techniques have also been explored, such as planar-curing methods. Will we see machines that promise even faster build times using radical ways such as having multiple light systems to cure portions of the resin in 3D? Will we see more controlled printing processes where different materials can be selectively channeled into an SLA build chamber to achieve multi-material or blended prints? Will we also see newer and smarter materials that behave according to multiple stimuli? Or perhaps even selective 4D disassembly, like the Blossom project by Richard Clarkson (Clarkson, 2014) who used different 3D printed materials to simulate a flower petal opening up in bloom? Clarkson's design consists of a hollow flexible chamber which is filled with air that inflates, causing each petal to react. But perhaps for now, time as the fourth dimension in $4 \mathrm{D}$ printing could refer to both paradigms - strategies that can be used to reduce the print process (such as through kinematics); and the use of smart materials where parts 
adapt themselves in response to the surrounding environment supporting the notion of selfassemblies. So 4D printing, whether fad or truly revolutionary, you decide, and time will tell!

\subsection{References}

Autodesk Inc. (2014), "Autodesk Research - Project Cyborg", available at: http://www.autodeskresearch.com/ projects/cyborg (accessed 1 February 2014)

Clarkson, R. (2014), "Blossom" available at: http://richardclarkson.com/blossom (accessed 1 February 2014)

Coelho M., Tibbits, S., Linder, N. and Reches, Y. (2013), "Hyperform - Folding Strategies for 3D Printing", available at: http://www.cmarcelo.com/hyperform (accessed 1 February 2014)

Corbett, J. (2012), "Reprap Colour Mixing Project", University of Bath, Faculty of Engineering and Design, Department of Mechanical Engineering, Final Year MEng Project.

Ge, Qi., Qi, H. Jerry and Dunn, Martin L., (2013), Applied Physics Letters Vol. 103 Iss. 131901 “Active materials by four-dimension printing"

Harms, B., Jung, H., Huang, V. and Chen, Y. (2012a), "Suspended Depositions" available at: http://nstrmnt.com/\#/suspended-depositions (accessed 1 February 2014)

Horne, R. (2012a), "3-way Quick-fit Extruder and Colour Blending Nozzle" available at: http://richrap.blogspot.co.uk/2012/08/3-way-quick-fit-extruder-and-colour.html (accessed 1 February 2014)

Maidin, S., Campbell, R.I. and Pei, E. (2012), "Development of a design feature database to support design for additive manufacturing", Assembly Automation, Vol. 32 Iss: 3, pp.235 - 244

Nervous System (2013), "Kinematics", available at: http://n-e-r-v-o-u-s.com/blog/?p=4467 (accessed 1 February 2014)

Pan, Y., Zhou, C. and Chen, Y. (2012), "Rapid Manufacturing in Minutes: the Development of a Mask Projection Stereolithography Process for High Speed Fabrication." ASME Journal of Manufacturing Science and Engineering, Vol. 134, No. 5

Stratasys (2013), "4D Printing Project", available at: http://www.stratasys.com/industries/education /4d-printing-project (accessed 1 February 2014)

Tibbits, S. (2012), Architectural Design Vol. 82 Iss: 68, pp.68 - 73

Tibbits, S. and Cheung, K. (2012), "Programmable materials for architectural assembly and automation", Assembly Automation, Vol. 32 Iss: 3, pp.216 - 225

Tibbits, S. and Olson, A. (2012a), "The Self-Assembly Line", available at: http://phyllotax.is/selfassembly (accessed 1 February 2014)

University of Pittsburgh, Swanson School of Engineering (2013), "Pitt-led research team receives grant to develop four-dimensional printing to create adaptive materials", available at: http://www.engineering. pitt.edu/News.aspx?id=2147508574 (accessed 1 February 2014) 
US Army webpage (2013), "Army 'can't afford' not to have Rapid Equipping Force, leader says", available at: http://www.army.mil/article/113454 (accessed 1 February 2014)

Wohler, T. (2013), Additive Manufacturing and 3D printing - State of the Industry Annual Worldwide progress Report 2014, Wohler's Associates, Inc

\subsection{Image References}

Figure 1: Stratasys (2014), “Objet500 Connex3 printer", available at: http://www.stratasys.com/3dprinters/design-series/precision/objet500-connex3\#content-slider-1 (accessed 1 February 2014)

Figure 2: Tibbits, S. and Olson, A. (2012a), "The Self-Assembly Line" TED Long Beach 2012 \& SEED Phyllotaxis Lab, available at: http://phyllotax.is/self-assembly (accessed 1 February 2014)

Figure 3: Nervous System (2013b), "Kinematics", Jessica Rosenkrantz and Jesse Louis-Rosenberg, available at http://n-e-r-v-o-u-s.com/blog/?p=4467 (accessed 1 February 2014)

Figure 4: Harms, B., Jung, H., Huang, V. and Chen, Y. (2012b), "Suspended Depositions" available at: http://nstrmnt.com/\#/suspended-depositions (accessed 1 February 2014)

Figure 5: Horne, R. (2012b), "3-way Quick-fit Extruder and Colour Blending Nozzle" available at: http://richrap.blogspot.co.uk/2012/08/3-way-quick-fit-extruder-and-colour.html (accessed 1 February 2014) 